Bull. Korean Math. Soc. 50 (2013), No. 3, pp. 935-949

http://dx.doi.org/10.4134/BKMS.2013.50.3.935

\title{
SURFACES IN $\mathbb{E}^{3}$ WITH $L_{1}$-POINTWISE 1-TYPE GAUSS MAP
}

\author{
Young Ho Kim and Nurettin Cenk Turgay
}

\begin{abstract}
In this paper, we study surfaces in $\mathbb{E}^{3}$ whose Gauss map $G$ satisfies the equation $\square G=f(G+C)$ for a smooth function $f$ and a constant vector $C$, where $\square$ stands for the Cheng-Yau operator. We focus on surfaces with constant Gaussian curvature, constant mean curvature and constant principal curvature with such a property. We obtain some classification and characterization theorems for these kinds of surfaces. Finally, we give a characterization of surfaces whose Gauss map $G$ satisfies the equation $\square G=\lambda(G+C)$ for a constant $\lambda$ and a constant vector $C$.
\end{abstract}

\section{Introduction}

The notion of finite type submanifolds and finite type mappings has been extremely studied since they were introduced by B.-Y. Chen in the late 1970's. Let $M$ be a submanifold of Euclidean space $\mathbb{E}^{m}$. A smooth mapping $\phi: M \rightarrow$ $\mathbb{E}^{N}$ is said to be of finite type if it can be expressed as a finite sum of eigenvectors of Laplace operator $\Delta$, that is, $\phi=\phi_{0}+\sum_{i=1}^{k} \phi_{i}$, where $\phi_{0}$ is a constant map, $\phi_{1}, \phi_{2}, \ldots, \phi_{k}$ non-constant maps such that $\Delta \phi_{i}=\lambda_{i} \phi_{i}, \lambda_{i} \in \mathbb{R}, i=1, \ldots, k$. More precisely, if the eigenvalues $\lambda_{1}, \lambda_{2}, \ldots, \lambda_{k}$ are distinct, then $\phi$ is said to be of $k$-type ([12]). Several results on the study of finite type mappings were summed up in a report by B.-Y. Chen ([10]).

The Gauss map of oriented submanifolds and isometric immersions from submanifolds into Euclidean space has been studied in several works in terms of being finite type (cf. $[6,8,9,13])$. For example, in [8, 9], finite type isometric immersions were studied. Note that if an isometric immersion $x$ from $M$ into $\mathbb{E}^{m}$ is of $k$-type, then the submanifold $M$ itself is said to be of $k$-type.

On the other hand, in [13], B.-Y. Chen and P. Piccinni studied compact submanifolds of Euclidean spaces with finite type Gauss map. If an oriented submanifold $M$ of a Euclidean space has 1-type Gauss map $G$, then $G$ satisfies

Received April 18, 2012.

2010 Mathematics Subject Classification. 53B25, 53C40.

Key words and phrases. Gauss map, $\square$-pointwise 1-type, Cheng-Yau operator.

The first author was supported by Basic Science Research Program through the National Research Foundation of Korea (NRF) funded by the Ministry of Education, Science and Technology (2010-0007184). 
$\Delta G=\lambda(G+C)$ for a constant $\lambda \in \mathbb{R}$ and a constant vector $C$. In time, it has been seen that the equation

$$
\Delta G=f(G+C)
$$

is satisfied for some function $f \in C^{\infty}(M)$ and constant vector $C$ by the Gauss map of some interesting submanifolds, such as a helicoid and a catenoid in $\mathbb{E}^{3}$ and several rotational surfaces in $\mathbb{E}^{4}([11,17])$. These submanifolds whose Gauss map $G$ satisfying (1.1) are said to have pointwise 1-type Gauss map. Several articles also appeared on submanifolds with such Gauss map (cf. [2, 3, $4,5,11,16,19,20])$.

Recently, in $[1,18]$ the definition of finite type submanifolds is extended in a natural way, by replacing the Laplace operator $\Delta$ with a sequence of operators $L_{0}, L_{1}, L_{2}, \ldots, L_{k}$ such that $L_{0}=-\Delta$. Let $L_{k}, k=1,2, \ldots, n-1$, denote the linearized operators of the first variation of the $(k+1)$ th mean curvature arising from normal variations of an hypersurface $M$ of the Euclidean space $\mathbb{E}^{n+1}$. Note that sometimes the letter $\square$ is used to denote the operator $L_{1}$ which is the Cheng-Yau operator introduced in [14]. By replacing the operator $\Delta=-L_{0}$ with the operator $L_{k}$ in the definition of finite type submanifold, the definition of being $L_{k}$-finite type has been given.

In this paper, we aim to extend the notion of pointwise 1-type Gauss map for the surfaces of Euclidean 3-space $\mathbb{E}^{3}$ in terms of the Chen-Yau operator $\square$. In Section 2, we give the definition of having $\square$-pointwise 1-type Gauss map with the basic definitions and the facts on the theory of surfaces in $\mathbb{E}^{3}$. In Section 3, we focus on the surfaces with constant Gaussian curvature, constant mean curvature and constant principal curvature. We obtain some classification and characterization theorems for such surfaces with $\square$-pointwise 1-type Gauss map. In Section 4, we give a characterization theorem for the surfaces of $\mathbb{E}^{3}$ with $\square$-(global) 1-type Gauss map.

The surfaces $M$ we are dealing with are smooth and connected unless otherwise stated.

\section{Preliminaries}

Let $M$ be an oriented surface in $\mathbb{E}^{3}$. We denote the Levi-Civita connections of $\mathbb{E}^{3}$ and $M$ by $\widetilde{\nabla}$ and $\nabla$, respectively and $D$ stands for the normal connection of $M$. Let $\left\{e_{1}, e_{2}\right\}$ be an orthonormal frame of tangent space of $M$ and $N$ the unit normal frame associated with the orientation of $M$. Then the Gauss and Weingarten formulas are given by

$$
\begin{aligned}
\widetilde{\nabla}_{e_{i}} e_{j} & =\nabla_{e_{i}} e_{j}+h_{i j} N, \\
\widetilde{\nabla}_{e_{i}} N & =-h_{i 1} e_{1}-h_{i 2} e_{2}, \quad i, j=1,2,
\end{aligned}
$$

where $h_{i j}$ are the components of the second fundamental form $h$ of $M$, i.e., $h_{i j}=h\left(e_{i}, e_{j}\right)$. 
The mapping

$$
\begin{aligned}
G: M & \rightarrow S^{2} \\
p & \mapsto N(p)
\end{aligned}
$$

is called the Gauss map of $M$ and the shape operator (or Weingarten map) $S$ of $M$ is defined as the differential of mapping $G$. Since $S$ is a linear self-adjoint operator, it has two real eigenvalues, called the principal curvatures of $M$ and the eigenvectors, called the principal directions of $M$, corresponding to these eigenvalues are orthogonal. Note that $S$ satisfies $S(X)=-\widetilde{\nabla}_{X} N$ for a tangent vector field $X$ of $M$.

Let $M$ be an oriented surface in $\mathbb{E}^{3}$ and $e_{1}, e_{2}$ the principal directions corresponding to the principal curvatures $k_{1}, k_{2}$, respectively. Then, we have $\widetilde{\nabla}_{e_{i}} N=-k_{i} e_{i}$ and $S=\operatorname{diag}\left(k_{1}, k_{2}\right)$. The (first) mean curvature $H$ and Gaussian (or the second mean) curvature $K$ of $M$ is defined as

$$
\begin{aligned}
H & =\frac{k_{1}+k_{2}}{2}, \\
K & =k_{1} k_{2} .
\end{aligned}
$$

If $H \equiv 0$ (resp., $K \equiv 0$ ), then $M$ is called minimal (resp., flat). We define the functions $\omega_{1}$ and $\omega_{2}$ as $\omega_{1}=\left\langle\nabla_{e_{1}} e_{1}, e_{2}\right\rangle$ and $\omega_{2}=-\left\langle\nabla_{e_{2}} e_{2}, e_{1}\right\rangle$, where $\langle$, denotes the inner product in $\mathbb{E}^{3}$.

On the other hand, the well-known Gauss and Codazzi equations become, respectively,

$$
\begin{array}{r}
e_{1}\left(\omega_{2}\right)-e_{2}\left(\omega_{1}\right)+\omega_{1}^{2}+\omega_{2}^{2}=-K, \\
e_{i}\left(k_{j}\right)=\omega_{j}\left(k_{1}-k_{2}\right)
\end{array}
$$

and the first and second structural equations are, respectively,

$$
\begin{aligned}
d \theta_{i} & =\theta_{i j} \wedge \theta_{j}, \\
d \theta_{12} & =-K \theta_{1} \wedge \theta_{2}
\end{aligned}
$$

for $i, j=1,2$ and $i \neq j$ where $\theta_{1}, \theta_{2}$ and $\theta_{i j}$ are the 1 -forms such that $\theta_{i}\left(e_{j}\right)=$ $\delta_{i j}$ and $\theta_{12}=\omega_{1} \theta_{1}+\omega_{2} \theta_{2}$ satisfying $\theta_{12}=-\theta_{21}$ and $\theta_{i i}=0$.

We will use $C^{\infty}\left(M, \mathbb{E}^{3}\right)$ to denote the space of all smooth functions from $M$ into the 3 -dimensional Euclidean space $\mathbb{E}^{3}$ and $C^{\infty}(M)$ will stand for the space of all smooth functions defined on $M$.

If $X \in C^{\infty}\left(M, \mathbb{E}^{3}\right)$ is tangent to $M$, its divergence $\operatorname{div} X$ is defined as the mapping which assigns every point $p$ at $M$ into trace of the linear mapping $Y(p) \mapsto\left(\nabla_{Y} X\right)(p)$. On the other hand, the gradient of a function $f \in C^{\infty}(M)$ is defined by $\nabla f=e_{1}(f) e_{1}+e_{2}(f) e_{2}$ and the Laplace operator acting on $M$ is $\Delta=\nabla_{e_{1}} e_{1}+\nabla_{e_{2}} e_{2}-e_{1} e_{1}-e_{2} e_{2}([15])$.

\subsection{Cheng-Yau operator}

Let $L_{k}: C^{\infty}(M) \rightarrow C^{\infty}(M), L_{k}(f)=\operatorname{tr}\left(P_{k} \circ \nabla^{2} f\right), k=1,2$ denote the linearized operator of the first variation of the $(k+1)$ th mean curvature arising from normal variations of the surface, where $P_{0}=I, P_{1}=\left(k_{1}+k_{2}\right) I-S$ and 
$I$ is the identity operator acting on the tangent bundle of $M$. Note that the operator $L_{0}$ is nothing but the Laplace operator acting on $M$, i.e., $L_{0}=-\Delta$ and $L_{1}=\square$ is called the Cheng-Yau operator introduced in [14].

As a matter of fact, it turns out to be $L_{k} f=\operatorname{div}\left(P_{k}(\nabla f)\right)$ for $f \in C^{\infty}(M)$ ([1]). By putting $k=1$ in this equation, we obtain

$$
\begin{aligned}
\square f & =\operatorname{div}\left(P_{1}(\nabla f)\right) \\
& =\operatorname{div}\left(k_{2} e_{1}(f) e_{1}+k_{1} e_{2}(f) e_{2}\right) \\
& =e_{1}\left(k_{2} e_{1}(f)\right)+e_{2}\left(k_{1} e_{2}(f)\right)-k_{1} \nabla_{e_{1}} e_{1}(f)-k_{2} \nabla_{e_{2}} e_{2}(f) .
\end{aligned}
$$

Hence, we have

$$
\square=e_{1}\left(k_{2}\right) e_{1}+e_{2}\left(k_{1}\right) e_{2}+k_{2}\left(e_{1} e_{1}-\nabla_{e_{2}} e_{2}\right)+k_{1}\left(e_{2} e_{2}-\nabla_{e_{1}} e_{1}\right) .
$$

\subsection{Definitions}

First, we extend the definition of operator $\square$ to the vector valued mappings defined on $M$ into Euclidean space $\mathbb{E}^{3}$ in a natural way below:

Definition 1. The operator $\square: C^{\infty}\left(M, \mathbb{E}^{3}\right) \rightarrow C^{\infty}\left(M, \mathbb{E}^{3}\right)$ is defined by

$$
\square Z=\left(\square z_{1}, \square z_{2}, \square z_{3}\right),
$$

where $z_{1}, z_{2}, z_{3} \in C^{\infty}(M)$ are the components of $Z$, i.e., $Z=\left(z_{1}, z_{2}, z_{3}\right)$.

Then, we have

$$
\square=e_{1}\left(k_{2}\right) \widetilde{\nabla}_{e_{1}}+e_{2}\left(k_{1}\right) \widetilde{\nabla}_{e_{2}}+k_{2}\left(\widetilde{\nabla}_{e_{1}} \widetilde{\nabla}_{e_{1}}-\widetilde{\nabla}_{\nabla_{e_{2}} e_{2}}\right)+k_{1}\left(\widetilde{\nabla}_{e_{2}} \widetilde{\nabla}_{e_{2}}-\widetilde{\nabla}_{\nabla_{e_{1}} e_{1}}\right) \text {. }
$$

By a direct calculation, we obtain

$$
\begin{aligned}
\square G= & e_{1}\left(k_{2}\right) \widetilde{\nabla}_{e_{1}} G+e_{2}\left(k_{1}\right) \widetilde{\nabla}_{e_{2}} G+k_{2}\left(\widetilde{\nabla}_{e_{1}} \widetilde{\nabla}_{e_{1}} G-\widetilde{\nabla}_{\nabla_{e_{2}} e_{2}} G\right) \\
& +k_{1}\left(\widetilde{\nabla}_{e_{2}} \widetilde{\nabla}_{e_{2}} G-\widetilde{\nabla}_{\nabla_{e_{1}} e_{1}} G\right) \\
= & -k_{1} e_{1}\left(k_{2}\right) e_{1}-k_{2} e_{2}\left(k_{1}\right) e_{2}+k_{2}\left(-\widetilde{\nabla}_{e_{1}}\left(k_{1} e_{1}\right)-\widetilde{\nabla}_{-\omega_{2} e_{1}} G\right) \\
& +k_{1}\left(-\widetilde{\nabla}_{e_{2}}\left(k_{2} e_{2}\right)-\widetilde{\nabla}_{\omega_{1} e_{2}} G\right) \\
= & -k_{1} e_{1}\left(k_{2}\right) e_{1}-k_{2} e_{2}\left(k_{1}\right) e_{2}+k_{2}\left(-e_{1}\left(k_{1}\right) e_{1}-k_{1} \omega_{1} e_{2}-k_{1}^{2} G-\omega_{2} k_{1} e_{1}\right) \\
& +k_{1}\left(-e_{2}\left(k_{2}\right) e_{2}+k_{2} \omega_{2} e_{1}-k_{2}^{2} G+\omega_{1} k_{2} e_{2}\right) .
\end{aligned}
$$

By re-arranging the last equation, we state:

Lemma 2.1. Let $M$ be an oriented surface in $\mathbb{E}^{3}$ with Gaussian curvature $K$ and mean curvature $H$. Then, the Gauss map $G$ of $M$ satisfies

$$
\square G=-\nabla K-2 H K G \text {. }
$$

Next we will give the definition concerning $\square$-pointwise 1-type Gauss map. 
Definition 2. An oriented surface $M$ of Euclidean space $\mathbb{E}^{3}$ is said to have $\square$-pointwise 1-type Gauss map if its Gauss map satisfies

$$
\square G=f(G+C)
$$

for a smooth function $f \in C^{\infty}(M)$ and a constant vector $C \in \mathbb{E}^{3}$. More precisely, a $\square$-pointwise 1-type Gauss map is said to be of the first kind if (2.12) is satisfied for $C=0$; otherwise, it is said to be of the second kind. Moreover, if (2.12) is satisfied for a constant function $f$, then we say $M$ has $\square$-(global) 1-type Gauss map.

We will use following lemma later:

Lemma 2.2. Let $M$ be an oriented surface in $\mathbb{E}^{3}$ and $e_{1}, e_{2}$ its principal directions corresponding to the principal curvatures $k_{1}, k_{2}$, respectively. A vector field $C \in C^{\infty}\left(M, \mathbb{E}^{3}\right)$ is constant if and only if

$$
\begin{aligned}
& e_{1}\left(C_{1}\right)=\omega_{1} C_{2}+k_{1} C_{3}, \\
& e_{1}\left(C_{2}\right)=-\omega_{1} C_{1}, \\
& e_{1}\left(C_{3}\right)=-k_{1} C_{1}, \\
& e_{2}\left(C_{1}\right)=\omega_{2} C_{2}, \\
& e_{2}\left(C_{2}\right)=-\omega_{2} C_{1}+k_{2} C_{3}, \\
& e_{2}\left(C_{3}\right)=-k_{2} C_{2},
\end{aligned}
$$

where $C_{1}=\left\langle C, e_{1}\right\rangle, C_{2}=\left\langle C, e_{2}\right\rangle, C_{3}=\langle C, G\rangle, \omega_{1}=\left\langle\nabla_{e_{1}} e_{1}, e_{2}\right\rangle$ and $\omega_{2}=$ $-\left\langle\nabla_{e_{2}} e_{2}, e_{1}\right\rangle$.

Proof. With the definitions above, we have $C=C_{1} e_{1}+C_{2} e_{2}+C_{3} G$. By a direct calculation we have

$$
\begin{aligned}
\widetilde{\nabla}_{e_{1}} C & =e_{1}\left(C_{1}\right) e_{1}+e_{1}\left(C_{2}\right) e_{2}+e_{1}\left(C_{3}\right) G+C_{1} \widetilde{\nabla}_{e_{1}} e_{1}+C_{2} \widetilde{\nabla}_{e_{1}} e_{2}+C_{3} \widetilde{\nabla}_{e_{1}} G \\
& =e_{1}\left(C_{1}\right) e_{1}+e_{1}\left(C_{2}\right) e_{2}+e_{1}\left(C_{3}\right) G+C_{1}\left(\omega_{1} e_{2}+k_{1} G\right)-C_{2} \omega_{1} e_{1}-C_{3} k_{1} e_{1} \\
& =\left(e_{1}\left(C_{1}\right)-\omega_{1} C_{2}-k_{1} C_{3}\right) e_{1}+\left(e_{1}\left(C_{2}\right)+\omega_{1} C_{1}\right) e_{2}+\left(e_{1}\left(C_{3}\right)+k_{1} C_{1}\right) G .
\end{aligned}
$$

Thus, $\widetilde{\nabla}_{e_{1}} C=0$ if and only if (2.13)-(2.15) hold. By a similar way, one can check that $\widetilde{\nabla}_{e_{2}} C=0$ is equivalent to $(2.16)-(2.18)$.

A function (or mapping) $\phi$ defined on $M$ is said to be harmonic if its Laplacian vanishes identically, i.e., $\Delta \phi=0$. By changing the Laplace operator $\Delta$ with Cheng-Yau operator $\square$, we give following definition:

Definition 3. An oriented surface $M$ of Euclidean space $\mathbb{E}^{3}$ is said to have $\square$-harmonic Gauss map if its Gauss map satisfies

$$
\square G=0 .
$$

The following theorem can be directly obtained from $[7$, p. 116, Proposition 3.1 by putting $c=0$. 
Theorem $2.3([7])$. Let $M$ be a surface in $\mathbb{E}^{3}$. $M$ has constant Gaussian curvature and constant mean curvature if and only if it is an open part of a sphere, a right circular cylinder $S^{1}(r) \times \mathbb{E}^{1}$ or a plane, where $S^{1}(r)$ denotes the circle with radius $r$.

\section{Some characterization theorems on surfaces with $\square$-pointwise 1-type Gauss map}

In this section, we will give some characterization theorems on the surfaces of $\mathbb{E}^{3}$ in terms of their Gauss map. We focus on the surfaces with constant mean curvature, constant Gaussian curvature or constant principal curvature.

\subsection{Surfaces with constant Gaussian curvature}

Theorem 3.1. An oriented surface $M$ in $\mathbb{E}^{3}$ has $\square$-harmonic Gauss map if and only if it is flat, i.e., its Gaussian curvature vanishes identically.

Proof. Because of Lemma 2.1, $M$ has $\square$-harmonic Gauss map if and only if $K$ is constant and $K H=0$. If $H=0$ and $K$ is constant, then Theorem 2.3 implies that $M$ is an open part of a plane. Hence, the proof is completed.

Theorem 3.2. An oriented surface $M$ in $\mathbb{E}^{3}$ has $\square$-pointwise 1-type Gauss map of the first kind if and only if it has constant Gaussian curvature.

Proof. The proof directly follows from Lemma 2.1.

Theorem 3.3. An oriented surface $M$ in $\mathbb{E}^{3}$ has $\square$-(global) 1-type Gauss map of the first kind if and only if it is either a flat surface or an open part of a sphere.

Proof. Because of Lemma 2.1 the Gauss map of $M$ satisfies $\square G=\lambda G$ for a constant $\lambda$ if and only if

$$
\nabla K=0 \quad \text { and } \quad-2 K H=\lambda .
$$

If $K=0$, then $\lambda=0$ and $M$ has $\square$-harmonic Gauss map. Thus, $M$ is flat because of Theorem 3.1. If $K \neq 0$, then $K$ and $H$ are constants. Therefore, Theorem 2.3 yields that $M$ is an open part of a sphere.

\subsection{Surfaces with constant mean curvature}

In this section, we first show that the only minimal surface with $\square$-pointwise 1-type Gauss map is plane. First, we state:

Corollary 3.4. An oriented surface $M$ in $\mathbb{E}^{3}$ has $\square$-pointwise 1-type Gauss map of the first kind if and only if it is an open part of a plane.

Proof. Proof directly follows from Theorem 2.3 and Theorem 3.2.

Next, we prove following proposition: 
Proposition 3.5. An oriented connected minimal surface $M$ in $\mathbb{E}^{3}$ has $\square$ pointwise 1-type Gauss map of the second kind if and only if it is an open part of a plane.

Proof. If $M$ has pointwise 1-type Gauss map of the second kind, then (2.12) is satisfied for a constant vector $C$ and a smooth function $f$. Let $\mathcal{O}=\{p \in$ $M \mid f(p) \neq 0\}$. We now suppose $\mathcal{O} \neq \emptyset$. Since $M$ is minimal, (2.11) and (2.12) imply $f(G+C)=-\nabla K$. Therefore, we have $C_{3}=\langle C, G\rangle=-1$ on $\mathcal{O}$. Thus, from (2.15) and (2.18) we obtain

$$
k_{1} C_{1}=k_{2} C_{2}=0
$$

on $\mathcal{O}$. We note that minimality of $M$ implies $k_{1}=-k_{2}$. Let $\mathcal{O}_{1}=\{p \in$ $\left.\mathcal{O} \mid k_{1}(p) \neq 0\right\}$. Then, $C_{1}=C_{2}=0$ on $\mathcal{O}_{1}$. Thus, the constant vector $C=-G$ on $\mathcal{O}_{1}$ and thus $\mathcal{O}_{1}$ is part of a plane which is a contradiction. Therefore, we have $\mathcal{O}_{1}=\emptyset$ which implies $k_{1}=k_{2} \equiv 0$ on $\mathcal{O}$. Hence, $\mathcal{O}$ is an open part of a plane. Moreover, by continuity, we have $M=\mathcal{O}$.

Conversely, suppose $M$ is an open part of a plane. Then, its Gauss map $G$ is a non-zero constant vector which implies $\square G=0$. Therefore, (2.12) is satisfied for $C=-G \neq 0$ and an arbitrary smooth function $f$. Hence, $M$ has $\square$-pointwise 1-type Gauss map of the second kind.

By combining Corollary 3.4 and Proposition 3.5, we obtain:

Theorem 3.6. An oriented minimal surface $M$ in $\mathbb{E}^{3}$ has $\square$-pointwise 1-type Gauss map if and only if it is an open part of a plane.

Next, we give a complete classification of surfaces with constant mean curvature whose Gauss map satisfying $\square G=\lambda(G+C)$ for a constant $\lambda$ and constant vector $C$.

Theorem 3.7. Let $M$ be a surface with constant mean curvature in $\mathbb{E}^{3}$. Then $M$ has $\square$-(global) 1-type Gauss map if and only if it is an open part of a sphere, a right circular cylinder or a plane.

Proof. Let $k_{1}$ and $k_{2}$ be the principal curvatures of $M$ and $e_{1}, e_{2}$ the corresponding principal directions. As $M$ has constant mean curvature, we have $k_{1}+k_{2}=h_{0}$ for a constant $h_{0}$ which implies

$$
e_{i}\left(k_{1}\right)=-e_{i}\left(k_{2}\right), \quad i=1,2 .
$$

Now, we suppose that $M$ has $\square$-(global) 1-type Gauss map. Then equation (2.12) is satisfied for $f=\lambda$ and a constant vector $C$, where $\lambda$ is a constant. Therefore, from (2.11) and (2.12) we obtain $-\nabla K-h_{0} K G=\lambda(G+C)$. From this equation, Codazzi equation (2.6) and (3.3) we get

$$
\begin{aligned}
\lambda C_{1} & =-e_{1}(K)=e_{1}\left(k_{1}\right)\left(k_{1}-k_{2}\right), \\
\lambda C_{2} & =-e_{2}(K)=e_{2}\left(k_{1}\right)\left(k_{1}-k_{2}\right), \\
\lambda\left(C_{3}+1\right) & =-h_{0} K .
\end{aligned}
$$


Moreover, since $C$ is a constant vector, equations (2.13)-(2.18) are satisfied because of Lemma 2.2.

By using (3.4) and (3.6) we obtain

$$
\lambda e_{1}\left(C_{3}+1\right)=-h_{0} e_{1}(K)=\lambda h_{0} C_{1} .
$$

Note that if $\lambda=0$, then we have $\square G=0$ and it implies that $M$ is flat because of Theorem 3.1. Thus $M$ is an open part of either a plane or a right circular cylinder because of Theorem 2.3. If $\lambda \neq 0$, then (3.7) implies $e_{1}\left(C_{3}\right)=h_{0} C_{1}$. This equation and (2.15) imply

$$
C_{1}\left(h_{0}+k_{1}\right)=0 .
$$

By a similar way, one can obtain

$$
C_{2}\left(h_{0}+k_{2}\right)=0 .
$$

Consider the open subset $\mathcal{O}=\left\{p \in M \mid k_{1} \neq-h_{0}, k_{2} \neq-h_{0}\right\}$ of $M$. Then, $C_{1}=C_{2}=0$ on $\mathcal{O}$. Thus, (3.4) and (3.5) imply that $K$ is constant on each component of $\mathcal{O}$. Thus, we have $C_{3}$ is a constant because of (3.6). If $C_{3}=0$, then $C=0$ which implies $M$ has constant Gaussian curvature because of Theorem 3.2. Therefore, $\mathcal{O}$ is an open part of a plane, a right circular cylinder or a sphere because of Theorem 2.3. On the other hand, if $C_{3} \neq 0$, then we have $G=C / C_{3}$ is a constant vector, from which, it follows that $\mathcal{O}$ is an open part of a plane. Moreover, in both cases continuity imply $\mathcal{O}=M$.

Now, we suppose that at every point of $M, C_{1} \neq 0$ or $C_{2} \neq 0$. Then, (3.8) and (3.9) imply that one of $k_{1}$ and $k_{2}$ is constant. Since $k_{1}+k_{2}$ is constant, $k_{1}$ and $k_{2}$ are constants and thus $M$ has constant Gaussian curvature. Hence, Theorem 2.3 implies it is an open part of a sphere, a right circular cylinder or a plane.

\subsection{Surfaces with constant principal curvature}

A surface in $\mathbb{E}^{3}$ is said to have a constant principal curvature if one of its principal curvatures is constant. In this subsection, we will give some characterization theorems for that kind of surfaces with $\square$-pointwise 1-type Gauss map.

Let $M$ be a surface in $M$ with the principal directions $e_{1}, e_{2}$ associated with the principal curvatures $k_{1}, k_{2}$, respectively. We suppose $k_{2}$ is constant. Then the Codazzi equation (2.6) become

$$
\begin{aligned}
e_{2}\left(k_{1}\right) & =\omega_{1}\left(k_{1}-k_{2}\right), \\
0 & =\omega_{2}\left(k_{1}-k_{2}\right) .
\end{aligned}
$$

(3.11) implies $k_{1}=k_{2}$ or $\omega_{2}=0$. Therefore, if $k_{1}-k_{2}$ does not vanish on an open set $\mathcal{O}$ of $M$, then from the Gauss equation (2.5) we have

$$
e_{2}\left(\omega_{1}\right)=\omega_{1}^{2}+K
$$

on $\mathcal{O}$. 
If $M$ has a constant principal curvature $k_{2}$ and $K=k_{1} k_{2}$ is a constant, then we have either $k_{2}=0$ or $k_{1}$ and $k_{2}$ are constants. Therefore, by using Theorem 2.3 and Theorem 3.2 we state:

Theorem 3.8. Let $M$ be a surface in $\mathbb{E}^{3}$ with a constant principal curvature. Then, $M$ has $\square$-pointwise 1-type Gauss map of the first kind if and only if it is either a flat surface or an open part of a sphere.

Next, we give the following theorem to classify surfaces with a constant principal curvature whose Gauss map satisfies $\square G=\lambda(G+C)$ for a constant $\lambda$ and a constant vector $C$ :

Theorem 3.9. Let $M$ be a surface with a constant principal curvature in $\mathbb{E}^{3}$. Then $M$ has $\square$-(global) 1-type Gauss map if and only if it is either a flat surface or an open part of a sphere.

Proof. Let $M$ be a surface with a constant principal curvature $k_{2}$ in $\mathbb{E}^{3}$ with $\square$-(global) 1-type Gauss map. Then equation (2.12) is satisfied for $f=\lambda$ and a constant vector $C$, where $\lambda$ is a constant. Therefore, (2.11) and (2.12) imply $-\nabla K-2 H K G=\lambda(G+C)$ from which and (3.10) we get

$$
\begin{aligned}
\lambda C_{1} & =-k_{2} e_{1}\left(k_{1}\right), \\
\lambda C_{2} & =-k_{2} \omega_{1}\left(k_{1}-k_{2}\right), \\
\lambda\left(C_{3}+1\right) & =-k_{1} k_{2}\left(k_{1}+k_{2}\right)
\end{aligned}
$$

as $k_{2}$ is constant.

Consider the open subset $\mathcal{U}=\left\{p \mid k_{1}(p) \neq 0\right.$ and $\left.k_{1}(p) \neq k_{2}\right\}$ and let $\mathcal{O}$ be a component of $\mathcal{U}$. Note that (3.11) implies $\omega_{2}=0$ on $\mathcal{O}$. Now, we assume that $k_{2} \neq 0$ and $\mathcal{O} \neq \emptyset$. If $\omega_{1}$ vanishes identically on an open part $\mathcal{O}_{2} \subset \mathcal{O}$, then we have $\theta_{12}=0$ and the second structural equation (2.8) implies that $\mathcal{O}_{2}$ is flat which is a contradiction. Therefore, without loss of generality, we may, locally, assume that $\omega_{1}$ does not vanish on $\mathcal{O}$. From (3.10) and (3.15) we get

$$
\lambda e_{2}\left(C_{3}\right)=-k_{2} \omega_{1}\left(k_{1}-k_{2}\right)\left(2 k_{1}+k_{2}\right) .
$$

Next, we use this equation and (3.14) in (2.18) to obtain

$$
k_{2} \omega_{1}\left(k_{1}-k_{2}\right)\left(2 k_{1}+k_{2}\right)=-k_{2}^{2} \omega_{1}\left(k_{1}-k_{2}\right),
$$

from which, we get $k_{1}=-k_{2}$ that implies $\mathcal{O}$ is minimal. Therefore, Theorem 3.6 implies that $\mathcal{O}$ is an open part of a plane, but this is a contradiction. Hence, we have either $\mathcal{O}=\emptyset$ or $k_{2}=0$. If $k_{2}=0$, then $M$ is flat. On the other hand, if $\mathcal{O}=\emptyset$, then $M$ is either flat or an open of a sphere.

The converse follows from Theorem 3.2.

Now suppose that $M$ is a non-flat surface which is not an open part of a sphere, which implies $k_{1} \neq k_{2}$ and $k_{2} \neq 0$. Thus from $(3.11)$ we get $\omega_{2}=0$. Therefore, we have $d \theta_{2}=0$ from the first structural equation (2.7) for $i=2, j=$ 1. Poincaré Lemma implies that $\theta_{2}$ is exact. Thus, there exist local coordinates $s, t$ such that the metric tensor of $M$ is of the form of $g=E^{2} d s^{2}+d t^{2}$ for a 
nowhere vanishing smooth function $E=E(s, t)$ which implies $e_{1}=\frac{1}{E} \partial_{s}$ and $e_{2}=\partial_{t}$. By a direct calculation together with the first structural equation (2.7) for $i=1, j=2$ we see that $\omega_{1}=-\frac{E_{t}}{E}$. Thus, we have $\theta_{12}=-E_{t} d s$. By using the second structural equation (2.8), we have $d \theta_{12}=E_{t t} d s \wedge d t=-K E d s \wedge d t$, from which we get $K=-\frac{E_{t t}}{E}$. On the other hand, from Codazzi equation (3.10) we obtain $k_{1}=\frac{k_{2} \phi_{1}}{E}+k_{2}$ for a smooth function $\phi_{1}=\phi_{1}(s)$. Thus, we have $K=-\frac{E_{t t}}{E}=\frac{k_{2}^{2} \phi_{1}}{E}+k_{2}^{2}$. Hence, we state following lemma:

Lemma 3.10. Let $M$ be a non-flat surface in $\mathbb{E}^{3}$ with a constant principal curvature $k_{2}$. Suppose that $M$ is not an open part of a sphere. Then there exists a local coordinate system $\{s, t\}$ such that the principal directions of $M$ are $e_{1}=\frac{1}{E} \partial_{s}, e_{2}=\partial_{t}$ and the corresponding principal curvatures satisfy

$$
k_{1}=\frac{k_{2} \phi_{1}}{E}+k_{2}
$$

for a smooth function $E=E(s, t)$ which is of the form of

$$
E=\phi_{2} \cos \theta-\phi_{1}, \quad \theta=k_{2} t+\phi_{3},
$$

where $\phi_{1}=\phi_{1}(s), \phi_{2}=\phi_{2}(s)$ and $\phi_{3}=\phi_{3}(s)$ are some smooth functions. Moreover, the Gaussian curvature of $M$ and the functions $\omega_{1}$ and $\omega_{2}$ are

$$
\begin{aligned}
K & =\frac{k_{2}^{2} \phi_{1}}{E}+k_{2}^{2}, \\
\omega_{1} & =-\frac{E_{t}}{E}
\end{aligned}
$$

and $\omega_{2}=0$.

By using Lemma 3.10, we obtain:

Theorem 3.11. Let $M$ be a surface with a constant principal curvature in $\mathbb{E}^{3}$. Then $M$ has $\square$-pointwise 1-type Gauss map if and only if it is either a flat surface or an open part of a sphere.

Proof. Let $M$ be a surface in $\mathbb{E}^{3}$ with $\square$-pointwise 1-type Gauss map and the principal curvatures $k_{1}, k_{2}$. We assume that $k_{2}$ is a constant. We now suppose $M$ is non-flat. Thus, we have $k_{2} \neq 0$. Let $\{s, t\}$ be the local coordinate system satisfying the conditions given in Lemma 3.10.

Since $M$ has $\square$-pointwise 1-type Gauss map, equation (2.12) is satisfied for a smooth map $f$ and a constant vector $C$. Therefore, (2.11) and (2.12) imply $-\nabla K-2 H K G=f(G+C)$ from which we obtain

$$
\begin{aligned}
f C_{1} & =\frac{1}{E} K_{s}, \\
f C_{2} & =-K_{t}, \\
f\left(C_{3}+1\right) & =-k_{1} k_{2}\left(k_{1}+k_{2}\right) .
\end{aligned}
$$

If $f$ is a constant function, Theorem 3.9 implies $M$ is either a flat surface or an open part of a sphere. Now, we assume that $f$ is not a constant function. 
On the other hand, as $C$ is a constant vector, Lemma 2.2 implies that $C_{1}$, $C_{2}$ and $C_{3}$ satisfy (2.13)-(2.18). As $\omega_{2}=0$ and $k_{2} \neq 0$, from (2.16)-(2.18) we obtain

$$
\begin{aligned}
& C_{1}=C_{1}(s), \\
& C_{2}=\psi_{1} \cos \rho, \\
& C_{3}=-\psi_{1} \sin \rho, \quad \rho=k_{2} t+\psi_{2},
\end{aligned}
$$

where $\psi_{1}=\psi_{1}(s)$ and $\psi_{2}=\psi_{2}(s)$ are some smooth functions.

On the other hand, by using (3.16), (3.18), (3.24) and (3.25) in (3.21) and (3.22) we obtain

$$
\begin{gathered}
f \psi_{1} \cos \rho=-\frac{k_{2}^{3} \phi_{2}}{E^{2}} \phi_{1} \sin \theta, \\
f\left(1-\psi_{1} \sin \rho\right)=-\frac{k_{2}^{3} \phi_{2}}{E^{2}} \cos \theta\left(\phi_{2} \cos \theta-\phi_{1}\right) .
\end{gathered}
$$

Next, we multiply both side of (3.26) and (3.27) by, respectively, $\left(1-\psi_{1} \sin \rho\right.$ ) and $\psi_{1} \cos \rho$ to get

$$
\psi_{1} \cos \rho \cos \theta\left(\phi_{2} \cos \theta-\phi_{1}\right)=\phi_{1} \sin \theta\left(1-\psi_{1} \sin \rho\right) .
$$

By a direct calculation, we see that this equation implies

$$
\frac{\psi_{1} \phi_{2}}{4}\left(\cos \left(2 \phi_{3}+\psi_{2}\right) \cos \left(3 k_{2} t\right)-\sin \left(2 \phi_{3}+\psi_{2}\right) \sin \left(3 k_{2} t\right)\right)+\mathcal{P}=0
$$

where $\mathcal{P}=\mathcal{P}(t, s)$ is of the form of $\mathcal{P}=A_{1} \cos \left(2 k_{2} t\right)+A_{2} \sin \left(2 k_{2} t\right)+A_{3} \cos \left(k_{2} t\right)$ $+A_{4} \sin \left(k_{2} t\right)$ for some smooth functions $A_{i}=A_{i}(s), i=1,2,3,4$. Since $\{\cos (m k t), \sin (m k t) \mid m \in \mathbb{N}\}$ is a set of linearly independent functions, (3.28) implies

$$
\psi_{1} \phi_{2}=0 \text {. }
$$

Consider the subset $\mathcal{U}=\left\{p \in M \mid \psi_{1}(p)=0\right\}$ of $M$ and let $\mathcal{O}$ be its interior. First, we assume that $\mathcal{O}=\emptyset$, that is $\psi_{1}=0$ on an open subset $M-\mathcal{U}$. Then, from (3.29) we have $\phi_{2}=0$ on $M-\mathcal{U}$. Thus, (3.16) and (3.17) imply $k_{1}=0$ on $M-\mathcal{U}$. Since $k_{1}$ is a continuous function, we have $k_{1}=0$ on $M$ which implies $M$ is flat, a contradiction.

Therefore, we have $\mathcal{O}=\operatorname{Int}(\mathcal{U}) \neq \emptyset$. On $\mathcal{O}, C_{2}=0$ from (3.24). Together with $(3.21)$, we get $K_{t}=0$. It is obvious that $K_{t}=0$ implies $K$ is constant because of (3.17) and (3.18). Hence, Proposition 3.8 implies that $\mathcal{O}$ is an open part of a sphere. By continuity, $\mathcal{O}$ must be $M$.

Converse is obvious. Hence the proof is completed.

\section{Surfaces with $\square$-1-type Gauss map}

In this section, we will obtain a necessary and sufficient condition for a surface to have $\square$-(global) 1-type Gauss map. Let $M$ be a non-flat surface in $\mathbb{E}^{3}$ with Gaussian curvature $K$, mean curvature $H$, the principal directions $e_{1}, e_{2}$ and the corresponding principal curvatures $k_{1}, k_{2}$. We assume that $M$ has $\square$-(global) 1-type Gauss map of the second kind. Then, Theorem 3.5 implies 
that $M$ is not minimal. Therefore, there exists an open set $\mathcal{U}$ of $M$ on which $H$ and $K$ are not vanishing. We may assume that $M=\mathcal{U}$.

Since $M$ has $\square$-(global) 1-type Gauss map, there exist a constant $\lambda \in \mathbb{R}$ and a constant vector $C \in \mathbb{E}^{3}$ such that $\lambda(G+C)=-\nabla K-2 K H G$ which implies

$$
\begin{aligned}
\lambda C_{1} & =-e_{1}(K), \\
\lambda C_{2} & =-e_{2}(K), \\
\lambda\left(C_{3}+1\right) & =-2 K H .
\end{aligned}
$$

Note that if $\lambda=0$, then we have $\square G=0$ which implies $M$ is flat because of Theorem (3.1). Thus, we have $\lambda \neq 0$.

Therefore, the vector $C$ is of the form of

$$
C=-\frac{e_{1}(K)}{\lambda} e_{1}-\frac{e_{2}(K)}{\lambda} e_{2}-\left(\frac{2 K H}{\lambda}+1\right) G .
$$

Since $C$ is a constant vector, its components $C_{1}, C_{2}$ and $C_{3}$ satisfy (2.13)-(2.18) because of Lemma 2.2. We put (3.4)-(3.6) on these equations to obtain

$$
\begin{aligned}
e_{1} e_{1}(K) & =\omega_{1} e_{2}(K)+k_{1}(2 K H+\lambda), \\
e_{1} e_{2}(K) & =-\omega_{1} e_{1}(K), \\
2 K e_{1}(H)+2 H e_{1}(K) & =-k_{1} e_{1}(K), \\
e_{2} e_{1}(K) & =\omega_{2} e_{2}(K), \\
e_{2} e_{2}(K) & =-\omega_{2} e_{1}(K)+k_{2}(2 K H+\lambda), \\
2 K e_{2}(H)+2 H e_{2}(K) & =-k_{2} e_{2}(K) .
\end{aligned}
$$

Note that a vector field $C$ given by (4.4) is constant if and only if (4.5)-(4.10) are satisfied.

After a direct calculation by using (2.6), (4.5) and (4.9), we obtain

$$
\begin{aligned}
-\Delta K & =2 H(2 K H+\lambda), \\
\square K & =2 K(2 K H+\lambda) .
\end{aligned}
$$

Therefore, we have

$$
\begin{aligned}
& \lambda=-\frac{\Delta K}{2 H}-2 K H, \\
& K \Delta K+H \square K=0 .
\end{aligned}
$$

On the other hand, from (4.6) we obtain

$$
e_{1} e_{2}(K)=\left(\nabla_{e_{1}} e_{2}\right) K .
$$

Next, from (4.7) and (4.10) we get

$$
\begin{aligned}
& e_{1}\left(k_{1}\right)\left(3 k_{1} k_{2}+k_{2}^{2}\right)+e_{1}\left(k_{2}\right)\left(2 k_{1}^{2}+2 k_{1} k_{2}\right)=0, \\
& e_{2}\left(k_{1}\right)\left(2 k_{2}^{2}+2 k_{1} k_{2}\right)+e_{2}\left(k_{2}\right)\left(k_{1}^{2}+3 k_{1} k_{2}\right)=0,
\end{aligned}
$$


respectively. By dividing both sides of (4.14) by $k_{2}^{2}$ and putting $k_{1}=\phi k_{2}$ we obtain

$$
\frac{(3 \phi+1) e_{1}(\phi)}{5 \phi^{2}+3 \phi}+\frac{e_{1}\left(k_{2}\right)}{k_{2}}=0
$$

from which, we obtain

$$
e_{1}\left(k_{1}^{1 / 3} k_{2}^{2 / 5}\left(5 k_{1}+3 k_{2}\right)^{4 / 15}\right)=0 .
$$

In a similar way, we get

$$
e_{2}\left(k_{1}^{2 / 5} k_{2}^{1 / 3}\left(3 k_{1}+5 k_{2}\right)^{4 / 15}\right)=0
$$

from (4.15). Hence, we have shown that if $M$ has $\square$-1-type Gauss map, then (4.12), (4.13), (4.16) and (4.17) are satisfied.

Conversely, let us assume that (4.12), (4.13), (4.16) and (4.17) are satisfied and $\lambda$ given by (4.11) is a constant. One can check that (2.12) is satisfied for $f=\lambda$ and the vector $C$ given by (4.4). We will show that $C$ is a constant vector.

It is obvious that (4.13) implies (4.6). Moreover, (4.6) implies (4.8) because of the identity $e_{1} e_{2}-e_{2} e_{1}=\nabla_{e_{1}} e_{2}-\nabla_{e_{2}} e_{1}$. On the other hand, (4.11) and (4.12) imply

$$
\begin{aligned}
-\Delta K & =\left(e_{1} e_{1}(K)-\omega_{1} e_{2}(K)\right)+\left(e_{2} e_{2}(K)+\omega_{2} e_{1}(K)\right)=2 H(2 K H+\lambda), \\
\square K & =k_{1}\left(e_{1} e_{1}(K)-\omega_{1} e_{2}(K)\right)+k_{2}\left(e_{2} e_{2}(K)+\omega_{2} e_{1}(K)\right)=2 K(2 K H+\lambda) .
\end{aligned}
$$

From these equations, one can obtain (4.5) and (4.9) under the condition $k_{1} \neq$ $k_{2}$. Note that if $k_{1}=k_{2}$ on an open subset of $M$, then $M$ becomes an open part of a sphere. Therefore, $K$ is constant and from (4.11) we have $\lambda=-2 K H$. Thus, equations (4.5) and (4.9) are still satisfied. On the other hand, by a direct calculation we see that (4.16) and (4.17) imply (4.7) and (4.10), respectively. Therefore, we have shown that if (4.5)-(4.10) are satisfied which implies the vector field $C$ given by (4.4) is constant. Hence, we state:

Proposition 4.1. Let $M$ be a surface in $\mathbb{E}^{3}$ with non-vanishing mean curvature $H$ and Gaussian curvature $K$. Then $M$ has $\square$-(global) 1-type Gauss map if and only if equations (4.12), (4.13), (4.16) and (4.17) are satisfied and $\lambda$ given by (4.11) is constant. In this case, (2.12) is satisfied for the constant function $f=\lambda$ and the constant vector $C \in \mathbb{E}^{3}$ given by (4.11) and (4.4).

Finally, we propose a problem.

Open Problem. Classify surfaces in $\mathbb{E}^{3}$ with $\square$-1-type Gauss map.

Acknowledgements. This work was done while the second named author was visiting Kyungpook National University, Korea in 2012. 


\section{References}

[1] L. J. Alias and N. Gürbüz, An extension of Takahashi theorem for the linearized operators of the higher order mean curvatures, Geom. Dedicata 121 (2006), 113-127.

[2] C. Baikoussis, Ruled submanifolds with finite type Gauss map, J. Geom. 49 (1994), no. $1-2,42-45$.

[3] C. Baikoussis and D. E. Blair, On the Gauss map of ruled surfaces, Glasgow Math. J. 34 (1992), no. 3, 355-359.

[4] C. Baikoussis, B.-Y. Chen, and L. Verstraelen, Ruled surfaces and tubes with finite type Gauss map, Tokyo J. Math. 16 (1993), no. 2, 341-349.

5] C. Baikoussis and L. Verstralen, The Chen-type of the spiral surfaces, Results Math. 28 (1995), no. 3-4, 214-223.

[6] D. D. Bleecker and J. L. Weiner, Extrinsic bounds on $\lambda_{1}$ of $\Delta$ on a compact manifold, Comment. Math. Helv. 51 (1976), no. 4, 601-609.

[7] B.-Y. Chen, Geometry of Submanifolds, Pure and Applied Mathematics, No. 22. Marcel Dekker, Inc., New York, 1973.

8] - On the total curvature of immersed manifolds. VI. submanifolds of finite type and their applications, Bull. Inst. Math. Acad. Sinica 11 (1983), no. 3, 309-328.

[9] - Total Mean Curvature and Submanifold of Finite Type, World Scientific, 1984.

[10] A report on submanifolds of finite type, Soochow J. Math. 22 (1996), no. 2, $117-337$.

[11] B.-Y. Chen, M. Choi, and Y. H. Kim, Surfaces of revolution with pointwise 1-type Gauss map, J. Korean Math. Soc. 42 (2005), no. 3, 447-455.

[12] B.-Y. Chen, J. M. Morvan, and T. Nore, Energy, tension and finite type maps, Kodai Math. J. 9 (1986), no. 3, 406-418.

[13] B.-Y. Chen and P. Piccinni, Submanifolds with finite type Gauss map, Bull. Austral. Math. Soc. 35 (1987), no. 2, 161-186.

[14] S. Y. Cheng and S. T. Yau, Hypersurfaces with constant scalar curvature, Math. Ann. 225 (1977), no. 3, 195-204.

[15] M. P. do Carmo, Riemannian Geometry, Translated from the second Portuguese edition by Francis Flaherty. Mathematics: Theory \& Applications. Birkhauser Boston, Inc., Boston, MA, 1992.

[16] U. Dursun, Hypersurfaces with pointwise 1-type Gauss map, Taiwanese J. Math. 11 (2007), no. 5, 1407-1416.

[17] U. Dursun and N. C. Turgay, General rotational surfaces in Euclidean space $\mathbb{E}^{4}$ with pointwise 1-type Gauss map, Math. Commun. (accepted).

[18] S. M. B. Kashani, On some $L_{1}$-finite type (hyper) surfaces in $\mathbb{R}^{n+1}$, Bull. Korean Math. Soc. 46 (2009), no. 1, 35-43.

[19] U. H. Ki, D. S. Kim, Y. H. Kim, and Y. M. Roh, Surfaces of revolution with pointwise 1-type Gauss map in Minkowski 3-space, Taiwanese J. Math. 13 (2009), no. 1, 317-338.

[20] Y. H. Kim and D. W. Yoon, Ruled surfaces with pointwise 1-type Gauss map, J. Geom. Phys. 34 (2000), no. 3-4, 191-205.

YOUNG Ho KIM

Department of Mathematics

KYUnGPOOK NATIONAL UNIVERSiTy

TAEgu 702-701, KoreA

E-mail address: yhkim@knu.ac.kr 
Nurettin Cenk Turgay

Department of Mathematics

Istanbul TeChNiCAL UnIVERSity

34469 Maslak, Istanbul, Turkey

E-mail address: turgayn@itu.edu.tr 